APS

physics

This is the accepted manuscript made available via CHORUS. The article has been published as:

\title{
Nontrivial Nature and Penetration Depth of Topological Surface States in SmB_\{6\} Thin Films
}

Tao Liu, Yufan Li, Lei Gu, Junjia Ding, Houchen Chang, P. A. Praveen Janantha, Boris Kalinikos, Valentyn Novosad, Axel Hoffmann, Ruqian Wu, C. L. Chien, and Mingzhong Wu Phys. Rev. Lett. 120, 207206 - Published 18 May 2018 DOI: 10.1103/PhysRevLett.120.207206 


\title{
Nontrivial Nature and Penetration Depth of Topological Surface States in $\mathrm{SmB}_{6}$ Thin Films
}

\author{
Tao Liu ${ }^{1}$, Yufan $\mathrm{Li}^{2}$, Lei Gu${ }^{3}$, Junjia Ding ${ }^{4}$, Houchen Chang ${ }^{1}$, P. A. Praveen Janantha ${ }^{1}$, Boris Kalinikos ${ }^{1,5}$, Valentyn \\ Novosad $^{4}$, Axel Hoffmann ${ }^{4}$, Ruqian $\mathrm{Wu}^{3}$, C. L. Chien ${ }^{2}$, and Mingzhong $\mathrm{Wu}^{1 \dagger}$ \\ ${ }^{1}$ Department of Physics, Colorado State University, Fort Collins, CO 80523, USA \\ ${ }^{2}$ Department of Physics and Astronomy, Johns Hopkins University, Baltimore, MD 21218, USA \\ ${ }^{3}$ Department of Physics and Astronomy, University of California, Irvine, CA 92697, USA \\ ${ }^{4}$ Materials Science Division, Argonne National Laboratory, Lemont, IL 60439, USA \\ ${ }^{5}$ St. Petersburg Electrotechnical University, 197376 St. Petersburg, Russia
}

\begin{abstract}
The nontrivial feature and penetration depth of the topological surface states (TSS) in $\mathrm{SmB}_{6}$ were studied via spin pumping. The experiments used $\mathrm{SmB}_{6}$ thin films grown on bulk magnetic insulator $\mathrm{Y}_{3} \mathrm{Fe}_{5} \mathrm{O}_{12}$ (YIG). Upon the excitation of magnetization precession in the YIG, a spin current is generated in the $\mathrm{SmB}_{6}$ that produces, via spin-orbit coupling, a lateral electrical voltage in the film. This spin-pumping voltage signal becomes considerably stronger as the temperature decreases from $150 \mathrm{~K}$ to $10 \mathrm{~K}$, and such an enhancement most likely originates from the spinmomentum locking of the TSS and may thereby serves as an evidence for the nontrivial nature of the TSS. The voltage data also show a unique film thickness dependence that suggests a TSS depth of $\sim 32 \mathrm{~nm}$. The spin-pumping results are supported by transport measurements and analyses using a tight binding model.
\end{abstract}

$\mathrm{SmB}_{6}$ is a Kondo insulator $(\mathrm{KI})^{1,2,3,4}$ that undergoes a metal-to-insulator transition when the temperature $(T)$ is reduced below $150 \mathrm{~K}^{5,6}$ This transition originates from hybridization between conduction electrons and localized $f$ electrons that opens a band gap at the Fermi level. ${ }^{7,8} \mathrm{SmB}_{6}$ has been studied extensively since 1969 when it was identified as the first KI. ${ }^{5}$ Since 2010, it has attracted a new wave of interest thanks to the prediction of nontrivial topology in the band structures of a group of KIs including $\mathrm{SmB}_{6}{ }^{9}$ It's predicted that at low $T$ some KIs could have a topologically protected metallic surface atop of an insulating bulk, manifesting a new class of materials - topological Kondo insulators. . $^{910,11,12,13,14}$

The prediction invoked considerable studies, experimental $^{15,16,17,18,19,20,21,22,23,24,25,26,27,28,29,30,31,32,33,34}$ and theoretical, ${ }^{9,10,11,12,13,35,36}$ on the topological surface states (TSS) in $\mathrm{SmB}_{6}$. These studies, mostly using bulk $\mathrm{SmB}_{6}$, seem to provide evidence for the existence of the TSS, but also yield controversial conclusions as exampled below. (1) The low- $T$ metallic surface states have been observed by transport, ${ }^{14,19,20,21}$ angle-resolved photoemission spectroscopy (ARPES), ${ }^{22,23,24,25}$ scanning-tunneling microscopy, ${ }^{26,27,28}$ neutron scattering, ${ }^{29}$ and de Hass-van Alphen measurments. ${ }^{30}$ The helical spin texture on the surfaces has also been observed using circular dichroism ARPES. ${ }^{31}$ Despite of those works, however, no Dirac cones have been found experimentally, leaving ample room for doubts about the nature of the observed surface states. Indeed, other ARPES studies ${ }^{32}$ suggested that the TSS in $\mathrm{SmB}_{6}$ could be topologically trivial states associated with an intrinsic property of hexaborides, while the helical spin texture observed ${ }^{31}$ might be attributed to spin-polarized Rashba-split states. (2) Torque magnetometry studies showed quantum oscillations in $\mathrm{SmB}_{6}$, but the data yielded conflicting results about whether the Fermi surface is two-dimensional ${ }^{30}$ or three-dimensional, ${ }^{33}$ only the former supporting the topological nature. On the other hand, magnetoresistance measurements did not find evidence of quantum oscillations. ${ }^{34}$ (3) Recent experiments showed that the low- $T$ limiting resistance in $\mathrm{SmB}_{6}$ slabs is independent of the slab thickness, as expected. ${ }^{14,19,20}$ This independence, however, could also arise from an accidental layer on the surface, ${ }^{14,37}$ and the penetration depth $\left(d_{\mathrm{TSS}}\right)$ of the TSS remains unknown.

$\mathrm{SmB}_{6}$ films with a thickness down to about $d_{\mathrm{TSS}}$ offer possibilities to address many of the unresolved debates. Further, $\mathrm{SmB}_{6}$ in thin film form enables device fabrication, allowing for exploration of potential applications. In contrast to considerable previous studies using bulk $\mathrm{SmB}_{6}$, work on thin films has been limited, mainly on film growth and characterization. ${ }^{38,39,40}$ Nevertheless, there have been reports on the superconducting proximity effect in $\mathrm{SmB}_{6} / \mathrm{Nb}$ bilayers ${ }^{41}$ and spin-orbit-torque switching in $\mathrm{SmB}_{6} / \mathrm{CoFeB}$ bilayers. ${ }^{42}$

This article reports the use of $\mathrm{SmB}_{6}$ films to study the nontrivial nature and depth of the TSS in $\mathrm{SmB}_{6}$. The experiments used 10-300 nm thick $\mathrm{SmB}_{6}$ films grown on $\mathrm{Y}_{3} \mathrm{Fe}_{5} \mathrm{O}_{12}$ (YIG) and spin pumping ${ }^{43,44,45,46,47}$ to probe the spin-momentum locking of the TSS. A microwave is used to excite spin waves in the YIG, and the latter pumps a spin current into the $\mathrm{SmB}_{6}$. Via spinorbit coupling (SOC) the spin current produces an 
electrical voltage across the $\mathrm{SmB}_{6}$ film, and the analysis of this signal enables the evaluation of the SOC strength.

The data show that in samples with $\mathrm{SmB}_{6}$ film thickness $d \leq 50 \mathrm{~nm}$, the spin-pumping voltage decreases monotonically when $T$ decreases from $300 \mathrm{~K}$ to $10 \mathrm{~K}$, similar to the responses in heavy metal (HM)/YIG. ${ }^{48,49}$ When $d \geq 80 \mathrm{~nm}$, however, the signal is surprisingly enhanced when $T$ is decreased over $10-150 \mathrm{~K}$. This enhancement likely reflects the low- $T$ enhancement of the SOC due to spin-momentum locking, demonstrating the nontrivial topological feature of the $\mathrm{SmB}_{6}$. At room temperature (RT), the $d$ dependence of the signal shows a behavior very similar to that in $\mathrm{HM} / \mathrm{YIG}^{47,50}$ and indicates a spin diffusion length of $\lambda_{\mathrm{sd}}=38.7 \pm 1.8$ nm. $^{47,51}$ The $d$ dependence at low $T$ is different and suggests $d_{\text {TSS }} \approx 32 \mathrm{~nm}$. The TSS are further examined by transport measurements and tight binding model-based analyses. $^{36}$

The experimental approach is depicted in Figs. 1(a) and $1(\mathrm{~b})$. The sample consists of $\mathrm{a} \mathrm{SmB}_{6}$ film grown by sputtering on a 0.5 -mm-thick YIG slab with a length $a=4 \mathrm{~mm}$ and a width $b=2 \mathrm{~mm}$. The stoichiometry of the $\mathrm{SmB}_{6}$ was confirmed by $\mathrm{X}$-ray photoelectron spectroscopy. ${ }^{42,52,53}$ The YIG is magnetized by a field $(H)$ along the $y$ axis. The magnetic moments in the YIG are driven to precess by placing a microstrip line on the sample and feeding it with a microwave. Via spin pumping, ${ }^{43,44,45,46,47,50}$ the precessing moments produce a spin current in the $\mathrm{SmB}_{6}$. This spin current is then converted into a charge current along the $x$ axis via SOC, through the inverse Edelstein effect ${ }^{46,54,55}$ or
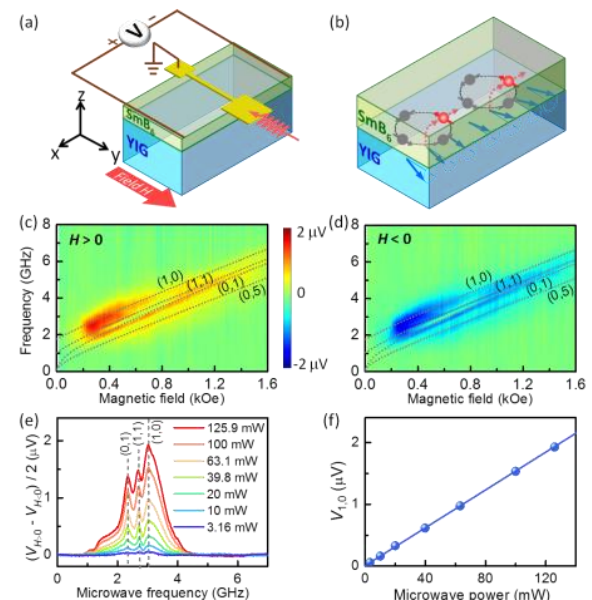

Fig. 1. Spin-pumping experiments. (a-b) Experiment approach. (c-d) Spin-pumping signals measured using opposite fields $(H)$ and an input microwave power $P_{\text {in }}=100$ $\mathrm{mW}$. The curves show calculated spin-wave frequencies. (e) Signals measured at different $P_{\text {in }}$, where $V_{H>0}\left(V_{H<0}\right)$ is the signal measured at $H=400 \mathrm{Oe}(H=-400 \mathrm{Oe})$. The vertical dashed lines indicate the calculated frequencies of three modes. (f) Amplitude of the $(1,0)$ peak in (e) vs. $P_{\text {in }}$. the inverse spin Hall effect ${ }^{43,44,45}$ depending on whether the TSS are present, resulting in a voltage signal $V$.

Figure 1(c) presents voltage signals measured for $H>0$ on a $\mathrm{SmB}_{6}(120 \mathrm{~nm}) / \mathrm{YIG}$ sample $(d=120 \mathrm{~nm})$. Strong signals present in some regions, and they are associated with standing spin-wave modes in the YIG. As the microwave magnetic field produced by the microstrip is spatially non-uniform, it excites spin waves in the YIG. $^{56}$ Those spin waves are quantized due to dimension confinement, with wavenumber vectors $^{57}$

$$
\mathbf{k}=\frac{m \pi}{a} \hat{\mathbf{x}}+\frac{n \pi}{b} \hat{\mathbf{y}}
$$

where $m$ and $n$ are mode indexes associated with the waves in the length and width directions, respectively. The analysis of those modes requires the development of a spin-wave dispersion relation for the YIG slab. As an approximation, the dispersion relation for thin films ${ }^{57,58}$ has been used to analyze the data. Four distinct modes have been identified, and the strongest signal appears over $0.2-0.7 \mathrm{kOe}$ and corresponds to the $(1,0)$ mode. $^{59}$

Figure 1(d) shows the data measured under the same conditions except $H<0$. The data show the almost same characteristics as those in Fig. 1(c) except an opposite voltage sign. This sign change is consistent with the facts that the YIG magnetization direction dictates the polarization of the spin currents, and a flip in the polarization leads to a sign change of $V \cdot{ }^{43,44,45}$ The signal might contain a circuit-associated background as well as contributions from non-magnetic effects (e.g. the Seebeck effect). ${ }^{60}$ These contributions are $H$-independent and can be easily removed by subtracting the signals measured using opposite fields.

The data in Figs. 1(c) and 1(d) were obtained at an input microwave power $P_{\text {in }}=100 \mathrm{~mW}$. To ensure that spin pumping is performed in a linear regime, the measurements using different $P_{\text {in }}$ are compared. Figure 1(e) shows the frequency-domain data $\left(V_{H>0}-V_{H<0}\right) /$ 2 obtained using different $P_{\text {in }}$. The $(1,0)$ mode is the strongest for all $P_{\text {in }}$, consistent with the data in Figs. 1(c) and 1(d). From the data in Fig. 1(e) one can plot the peak voltage of the $(1,0)$ mode, $V_{1,0}$, as a function of $P_{\text {in }}$, as in Fig. 1(f). The data in Fig. 1(e) show that the peak frequencies do not change with $P_{\text {in }}$, while those in Fig. 1(f) show linear behavior, suggesting that measurements involve neither microwave heating nor nonlinear spin pumping. The data below were taken at $P_{\text {in }}=100 \mathrm{~mW}$.

The above-described approach facilitates the use of spin pumping to explore the TSS in $\mathrm{SmB}_{6}$. The key point is that when nontrivial TSS are present, the spinmomentum locking enhances $\mathrm{SOC}$ in $\mathrm{SmB}_{6}$ and thereby results in strong voltage signals. 
Figure 2 presents the spin-pumping data for $\mathrm{SmB}_{6}(20 \mathrm{~nm}) / \mathrm{YIG}$ (top row) and $\mathrm{SmB}_{6}(120 \mathrm{~nm}) / \mathrm{YIG}$ (bottom row). For $\mathrm{SmB}_{6}(20 \mathrm{~nm}) / \mathrm{YIG}$, the signal diminishes as $T$ decreases from $297 \mathrm{~K}$ to $10 \mathrm{~K}$. This is mainly because the damping in polycrystalline YIG becomes stronger at low $T$ due to the enhancement in porosity- and anisotropy-associated two-magnon scattering. ${ }^{61}$ The larger damping results in weaker spin waves in the YIG and consequently a smaller spin current in the $\mathrm{SmB}_{6}$. In contrast, $\mathrm{SmB}_{6}(120 \mathrm{~nm}) / \mathrm{YIG}$ shows different behavior - the signal becomes weaker as $T$ is reduced from $297 \mathrm{~K}$ to $150 \mathrm{~K}$, but a further decrease in $T$ leads to a substantial increase in the signal. This enhancement is attributed to the TSS, as discussed below. The data also indicate that with decreasing $T$, the peaks shift to higher frequencies. This shift results from the increase of magnetization with decreasing $T^{62,63}$

Similar measurements were performed on other samples with different $d$. The data were analyzed with two approaches. One uses the amplitude of the main mode, $V_{1,0}$, to represent the signal strength and examines how $V_{1,0}$ varies with $T$ and $d$. The other examines the area $(A)$ under the $V$ profile and explores its $T$ and $d$ dependences. The $V_{1,0}$ data are given in Fig. 3. The $A$ data (see Fig. S3) show the same results as the $V_{1,0}$ data.

Figure 3(a) presents $V_{1,0}(T)$ for nine samples. Obviously, the signals from different samples show different $T$ dependences. To better present this, Fig. 3(b) gives the $V_{1,0}$ values normalized by that measured at $T=297$ K. Three observations are evident in Fig. 3(b). (1) For samples with $d \leq 50 \mathrm{~nm}$, the ratio " $V_{1,0} / V_{1,0}(297 \mathrm{~K})$ " decreases monotonically as $T$ decreases. (2) For the one with $d=80 \mathrm{~nm}$, the ratio drops when $T$ decreases from $297 \mathrm{~K}$ to $150 \mathrm{~K}$, but surprisingly increases as $T$ is further lowered. (3) For those with $d \geq 100$, the ratio decreases first, just like in

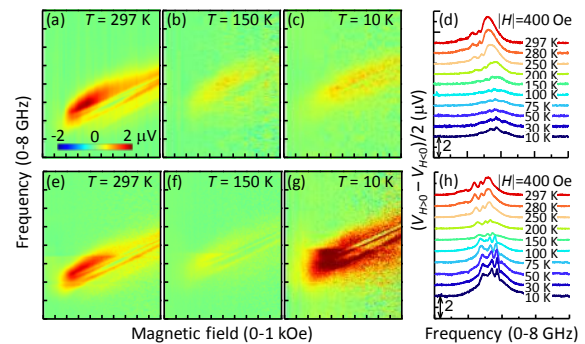

Fig. 2. Evidence for nontrivial TSS. The first and second rows present the data for $\mathrm{SmB}_{6}(20 \mathrm{~nm}) / \mathrm{YIG}$ and $\mathrm{SmB}_{6}(120 \mathrm{~nm}) / \mathrm{YIG}$, respectively. In each row, the color maps present the signals measured for $H>0$ at different $T$, while the rightmost diagram shows the $\left(V_{H>0}-V_{H<0}\right) / 2$ signals measured for $|H|=0.4 \mathrm{kOe}$ at different $T$. All the color maps use the same scale shown by the color bar in (a). thinner samples, but then increases substantially. As $T$ changes from $150 \mathrm{~K}$ to $10 \mathrm{~K}$, the ratio is enhanced by a factor of 4.1 for $\mathrm{SmB}_{6}(120 \mathrm{~nm}) / \mathrm{YIG}$ and a factor of 5.4 for $\mathrm{SmB}_{6}(300 \mathrm{~nm}) / \mathrm{YIG}$.

The results from Figs. 2, 3(a), and 3(b), together with those from Figs. S3 and S8, provide strong evidence for the nontrivial nature of the TSS in $\mathrm{SmB}_{6}$. Specifically, due to damping enhancement in YIG at low $T,{ }^{61}$ a decrease in $T$ leads to weaker spin pumping and accordingly a weaker voltage signal. As a result, the signal intensity in HM/YIG decreases monotonically with decreasing $T$, as shown in Fig. S8 and reported previously. ${ }^{48,49}$ Note that SOC in HM is insensitive to $T$ and is therefore not responsible for the monotonic voltage decrease. For $\mathrm{SmB}_{6} / \mathrm{YIG}$ samples, the signal intensity also decreases when $T$ is reduced from $297 \mathrm{~K}$ to $\sim 150 \mathrm{~K}$, as shown in Figs. 3(b) and S3(b). However, when $T$ is further reduced, in samples with $d \geq 80 \mathrm{~nm}$ the TSS start to be present and the spinmomentum locking start to enhance SOC, resulting in low- $T$ signal enhancement. Without the spinmomentum locking, one would expect a monotonic $T$ dependence just as in HM/YIG. Indeed, the signal does decrease with decreasing $T$ in samples with $d \leq 50 \mathrm{~nm}$ in which the nontrivial TSS are absent because the films are so thin that the top and bottom surface states overlap (see Figs. 5(a) and 5(b)). Note that the low- $T$ enhancement starting from $\sim 150 \mathrm{~K}$ is consistent with previous observations in which the TSS start to appear at $\sim 150 \mathrm{~K}$ and become stronger with a further decrease in $T^{23,24}$

The data in Fig. 3(b) also suggest notable $d$ dependences. To better show this, the $V_{1,0}$ data for $T=297 \mathrm{~K}$ and $T=10 \mathrm{~K}$ are plotted as a function of $d$ in
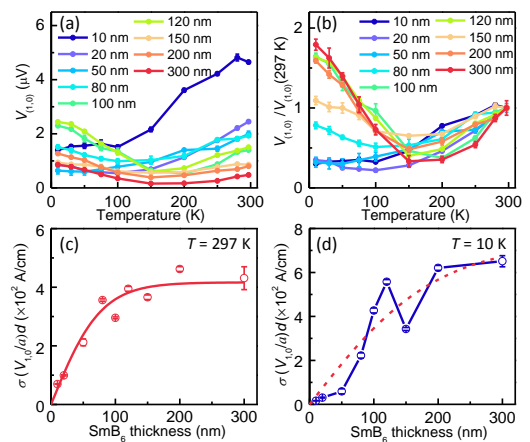

Fig. 3. Temperature and length characteristics of TSS. (a) Amplitude of the $(1,0)$ mode, $V_{1,0}$, as a function of $T$ for nine samples with different $d$. (b) The same $V_{1,0}$ data in (a) but normalized by the $V_{1,0}$ values at $T=297 \mathrm{~K}$. (c) and (d) give the $\sigma\left(V_{1,0} / a\right) d$ data at $297 \mathrm{~K}$ and $10 \mathrm{~K}$, respectively, as a function of $d$, with the error bars estimated from the noise level of the signals. The red curves in (c) and (d) are fits to Eq. (2). 
Figs. 3(c) and 3(d). The vertical axis shows $\sigma \frac{V_{1,0}}{a} d(\sigma$ $\mathrm{SmB}_{6}$ conductivity), which represents the signal strength that is independent of the sample dimensions and the resistivity. The red curves show fits to ${ }^{47,50}$

$$
\sigma \frac{V_{1,0}}{a} d=C \lambda_{\mathrm{sd}} \tanh \left(\frac{d}{2 \lambda_{\mathrm{sd}}}\right)
$$

which has been widely used to evaluate the spinpumping signals in HM/ferromagnet. ${ }^{64}$

The data in Fig. 3(c) can be fitted nicely. This is expected as $\mathrm{SmB}_{6}$ is known to have no TSS and show metallic behavior at RT. The saturation response is typical in $\mathrm{HM} /$ ferromagnet $^{47,50}$ and is not due to the electromagnetic shielding of the $\mathrm{SmB}_{6}$ (see the Supplementary Information $\left.{ }^{65,66}\right)$. The fitting yields $\lambda_{\mathrm{sd}}=38.7 \pm 1.8 \mathrm{~nm}$, which is longer than in HM..$^{50,51}$

Interestingly, the data in Fig. 3(d) cannot be fitted by Eq. (2). In fact, over $d=10-100 \mathrm{~nm}$ the experimental response shows a clear positive curvature, opposite to the expectation of Eq. (2). The different features indicated by the data in Figs. 3(c) and 3(d) suggest that $\mathrm{SmB}_{6}$ behaves like a HM at RT but differs from HMs at low $T$. This can be attributed to the presence of the TTS at low $T$. Specifically, at RT, as the TSS are absent in $\mathrm{SmB}_{6}$ and the $\mathrm{SOC}$ is similar to that in HMs, the $d$ dependence for $\mathrm{SmB}_{6}$ is expected to be similar to that for HMs; at low $T$, since the nontrivial TSS are absent in very thin $\mathrm{SmB}_{6}$ films but are present in thicker films, one would expect a jump in the " $V_{1,0}$ vs. $d$ " response that cannot be described by Eq. (2). The data in Fig. 3(d) indeed show a jump response. When $d$ increases, the signal strength increases slightly over $d=10-50 \mathrm{~nm}$ but starts to rise sharply at $d=50-80 \mathrm{~nm}$, suggesting $d_{\mathrm{TSS}}=25-40 \mathrm{~nm}$ or $\sim 32 \mathrm{~nm}$. In samples with $d>2 d_{\mathrm{TSS}}$, the TSS at the top and bottom surfaces of the $\mathrm{SmB}_{6}$ film are decoupled and show a nontrivial feature, resulting in enhanced SOC. When $d<2 d_{\mathrm{TSS}}$, the wave functions of the two surfaces overlap, suppressing the TSS.

Transport measurements were conducted on all nine samples. Figure 4(a) gives representative resistance $R(T)$. For both $\operatorname{SmB}_{6}(100 \mathrm{~nm}) / \mathrm{YIG}$ and $\mathrm{SmB}_{6}(300 \mathrm{~nm}) / \mathrm{YIG}$, with decreasing $T, R$ increases monotonically first and then reaches a plateau at $\sim 10 \mathrm{~K}$. $\mathrm{SmB}_{6}(20 \mathrm{~nm}) / \mathrm{YIG}$ shows a very similar response, except that the plateau is not as flat as those of the other two. These results are consistent with previous reports. ${ }^{67,68}$ The increase of $R$ with decreasing $T$ is a common feature of KIs and results from the gap opening due to hybridization between conduction electrons and $f$ electrons, while the low- $T$ plateau may be associated with the TSS. ${ }^{14}$ With decreasing $T$, the TSS start to present at $\sim 150 \mathrm{~K},{ }^{23,24}$ but the thermal excited bulk conductivity does not reduce to be a very small value till $\sim 10 \mathrm{~K}$. The overall $R$ increase is close to that reported previously for $\mathrm{SmB}_{6}$ films, ${ }^{40,41,42}$ but is notably smaller than in bulk $\mathrm{SmB}_{6} \cdot{ }^{14,67,68}$ This difference is likely because defects in polycrystalline films may serve as additional conducting channels, while the density of defects is low in $\mathrm{SmB}_{6}$ singlecrystals.

One can perform more quantitative analyses by plotting the conductance $G(=1 / R)$ vs. $T$, as in Fig. 4(b), and modeling $G$ as the combination of two contributions - one deceases with decreasing $T$ and goes to zero at $T=0$, while the other is $T$-independent residual conductance, ${ }^{20,41,68,69}$

$$
G=G_{b} e^{-\frac{E_{g}}{k_{B} T}}+G_{r}
$$

The exponential term describes the bulk conductivity change due to the gap opening. $G_{b}$ is the bulk conductance in the high $T$ limit. $E_{g}$ is the gap energy. Defining $\sigma_{0}$ as the high- $T$ bulk conductivity limit, $G_{b}$ can be evaluated as $\sigma_{0} \frac{w}{l}\left(d-2 d_{\mathrm{TSS}}\right)(w-$ sample width; $l$-sample length). $G_{r}$ is the residual conductance, consisting of a metallic surface contribution and a defect-associated, $T$-independent bulk contribution.

The curves in Fig. 4(b) show fits to Eq. (3). The fits are good for all three samples. The fitting-yielded $E_{g}, G_{b}$, and $G_{r}$ are presented in Figs. 4(c), 4(d), and 4(e), respectively. In Fig. 4(c), the dashed lines indicate $E_{g}$ values reported previously for $\mathrm{SmB}_{6}$ bulks ${ }^{20,68,69}$ and films. ${ }^{41}$ The fitting-yielded $E_{g}$ values are close to each other, suggesting no $d$ dependence, and are also consistent with the previous values. $G_{b}$ increases with $d$, with a kink at $d \approx 120 \mathrm{~nm}$. The linear fitting of the five points on the right side of the kink to $\sigma_{0} \frac{w}{l}(d-$ $2 d_{\mathrm{TSS}}$ ) yielded $d_{\mathrm{TSS}} \approx 31 \mathrm{~nm}$, which is surprisingly close to that from spin-pumping measurements. The fitting also yielded $\sigma_{0}=2.7 \times 10^{4} \Omega^{-1} \cdot \mathrm{m}^{-1}$, which is almost the

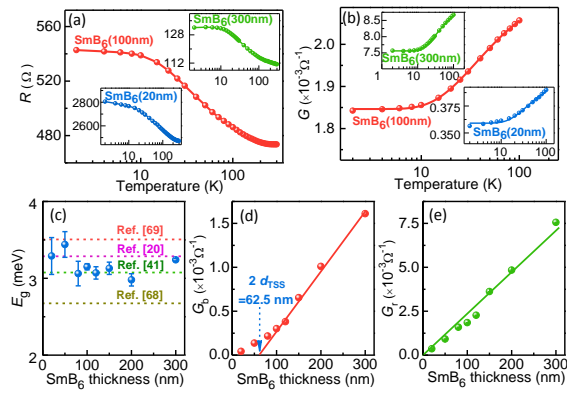

Fig. 4. Transport measurements confirming $\mathrm{SmB}_{6}$ film quality and TSS depth. (a) Resistance $R$ vs. T. The curves are guides for eyes. (b) Conductance $G$ vs. $T$. The curves are fits to Eq. (3). (c), (d), and (e) present the fitting-yielded gap energy $E_{\mathrm{g}}$, bulk conductance $G_{\mathrm{b}}$, and residual conductance $G_{\mathrm{r}}$, respectively. The lines in (c) indicate the values reported previously. The line in (d) is a liner fit to the five points on the right side of the kink. The line in (e) is a linear fit. 
same as that for bulk $\mathrm{SmB}_{6}{ }^{68,69}$ The data in Fig. 4(e) show an approximately linear response, indicating that the defect-associated conductivity dominates over the metallic surface contribution. The existence of the low$T R$ plateaus, the nice fitting of $G(T)$, and the agreement of $E_{g}$ and $\sigma_{0}$ with the values for single-crystal $\mathrm{SmB}_{6}$ together confirm the high quality of the $\mathrm{SmB}_{6}$ films.

The spin-pumping results were further supported by tight binding model-based analyses. ${ }^{36,70,71,72}$ Figure 5 shows main results. The interaction between the top and bottom surface states across a $21-\mathrm{nm}$ film is very strong, as manifested by the gap opening for the surface states shown in (a) and the long-range distribution of the wave functions in (b). As $d$ increases, the two surface states eventually decouple and the gap closes, as displayed by the results for the $83-\mathrm{nm}$ film. With this band structure, the spin-momentum locking feature is recovered and produces the low- $T$ enhancement of the spin-pumping signal. Further simulations indicate $d_{\mathrm{TSS}}=30-40 \mathrm{~nm}$, consistent with the experimental values.

Previous studies showed $d_{\mathrm{TSS}} \approx 16 \mathrm{~nm}^{39}$ and $d_{\text {TSS }} \approx 6 \mathrm{~nm},{ }^{41}$ which are smaller than the abovediscussed values. The reason for this is currently unknown and may be due to different qualities of the $\mathrm{SmB}_{6}$ films, but it should be mentioned that the values were determined through transport measurements only, with no evidence of the nontrivial nature of the TSS provided. All of those $d_{\text {TSS }}$ values are larger than

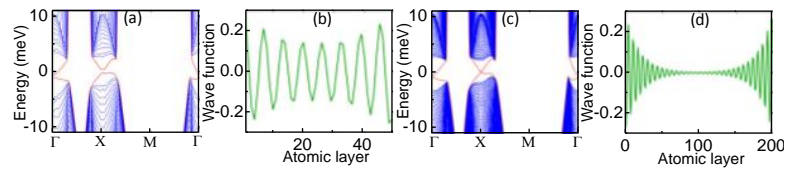

Fig. 5. Theoretical analysis of TSS in $\mathbf{S m B}_{6}$. (a) and (b) present the band structure and wave function of the surface state of a 50-layer film (21 nm), respectively; while (c) and (d) present the results of a 200-layer film $(83 \mathrm{~nm})$.

prototypical topological insulators. ${ }^{73}, 74$ This is understandable as $d_{\mathrm{TSS}}$ is roughly proportional to $1 / E_{g}$ (Fig. S6(b)). Spin-pumping experiments were previously conducted on a structure consisting of a Permalloy film grown on a $\mathrm{SmB}_{6}$ bulk. ${ }^{75}$ The signals were limited to very low $T(2 \mathrm{~K})$, inconsistent with the expected $T$ dependence of the TSS in $\mathrm{SmB}_{6}{ }^{23,24}$ The use of bulk $\mathrm{SmB}_{6}$ and the signals measurable only at low $T$ make it impossible to determine whether the TSS is responsible for the spin-to-charge conversion and what $d_{\text {TSS }}$ is. $^{76}$

In summary, spin-pumping measurements have been conducted on $\mathrm{SmB}_{6} / \mathrm{YIG}$. The low- $T$ spinpumping signal enhancement likely provides evidence for the nontrivial feature of the TSS in $\mathrm{SmB}_{6}$, while the $d$ dependence of the signals indicates $d_{\mathrm{TSS}} \approx 31 \mathrm{~nm}$ and $\lambda_{\mathrm{sd}} \approx 39 \mathrm{~nm}$. The results were supported by transport measurements and theoretical analyses. 
${ }^{1}$ G. Aeppli, and Z. Fisk, Comm. Condens. Matter Phys. 16, 155 (1992).

${ }^{2}$ Z. Fisk et al., Physica B 223, 409 (1996).

${ }^{3}$ P. S. Riseborough, Adv. Phys. 49, 257 (2000).

${ }^{4}$ P. Coleman, Heavy fermions: Electrons at the edge of magnetism. Handbook of Magnetism and Advanced Magnetic Materials 1, 95-148 (Wiley, 2007).

${ }^{5}$ A. Menth, E. Buehler, and T. H. Geballe, Phys. Rev. Lett. 22, 295 (1969).

${ }^{6}$ L. M. Falicov, and J. C. Kimball, Phys. Rev. Lett. 22, 997 (1969).

${ }^{7}$ H. Tsunetsugu, M. Sigrist, and K. Ueda, Rev. Mod. Phys. 69, 809 (1997).

${ }^{8}$ M. Dzero, J. Xia, V. Galitski, and P. Coleman, Annu. Rev. Condens. Matter Phys. 7, 249 (2016).

${ }^{9}$ M. Dzero, K. Sun, V. Galitski, and P. Coleman, Phys. Rev. Lett. 104, 106408 (2010).

${ }^{10}$ T. Takimoto, J. Phys. Soc. Jpn. 80, 123710 (2011).

${ }^{11}$ M. Dzero, K. Sun, P. Coleman, and V. Galitski, Phys. Rev. B 85, 045130 (2012).

${ }^{12}$ V. Alexandrov, M. Dzero, and P. Coleman, Phys. Rev. Lett. 111, 226403 (2013).

${ }^{13}$ F. Lu, J. Zhao, H. Weng, Z. Fang, and X. Dai, Phys. Rev. Lett. 110, 096401 (2013).

${ }^{14}$ D. J. Kim, J. Xia, and Z. Fisk, Nat. Mater. 13, 466 (2014).

${ }^{15}$ Y. Nakajima, P. Syers, X. Wang, R. Wang, and J. Paglione, Nat. Phys. 12, 213 (2015).

${ }^{16}$ A. Stern, D. K. Efimkin, V. Galitski, Z. Fisk, and J. Xia, Phys. Rev. Lett. 116, 166603 (2016).

${ }^{17}$ A. Stern, M. Dzero, V. M. Galitski, Z. Fisk, and J. Xia, Nat. Mater. 16, 708 (2017).

${ }^{18}$ P. K. Biswas et al., Phys.Rev. B 95, 020410(R) (2017).

${ }^{19}$ D. J. Kim et al., Sci. Rep. 3, 3150 (2013).

${ }^{20}$ P. Syers, D. Kim, M. S. Fuhrer, and J. Paglione, Phys. Rev. Lett. 114, 096601 (2015).

${ }^{21}$ W. A. Phelan et al., Phys. Rev. X 4, 031012 (2014).

${ }^{22}$ M. Neupane et al., Nat. Commun. 4, 2991 (2013).

${ }^{23}$ J. Jiang et al., Nat. Commun. 4, 3010 (2013).

${ }^{24}$ N. Xu et al., Phys. Rev. B 88, 121102 (2013).

${ }^{25}$ J. D. Denlinger et al., Preprint at http://arxiv.org/abs/1312.6637.

${ }^{26}$ W. Ruan et al., Phys. Rev. Lett. 112, 136401 (2014).

${ }^{27}$ S. Rößler et al., Proc. Natl. Acad. Sci. U.S.A. 111, 4798 (2014).

${ }^{28}$ M. M. Yee et al., Preprint at https://arxiv.org/abs/1308.1085.

${ }^{29}$ W. T. Fuhrman et al., Phys. Rev. Lett. 114, 036401 (2015).

${ }^{30}$ G. Li et al., Science 346, 1208 (2014).

${ }^{31}$ N. Xu et al., Nat. Commun. 5, 4566 (2014).

${ }^{32} \mathrm{P}$. Hlawenka et al., Preprint at https://arxiv.org/abs/1502.01542.

${ }^{33}$ B. S. Tan et al., Science 349, 287 (2015).

${ }^{34}$ S. Thomas et al., Phys. Rev. B 94, 205114 (2016).

${ }^{35}$ V. Alexandrov, P. Coleman, and O. Erten, Phys. Rev. Lett. 114, 177202 (2015). 
${ }^{36}$ P. P. Baruselli, and M. Vojta, Phy. Rev. B 93, 195117 (2016).

${ }^{37}$ K. von Klitzing, and G. Landwehr, Solid State Commun. 9, 2201 (1971).

${ }^{38}$ H. Shishido, Y. Yoneda, T. Yoshida, S. Noguchi, and T. Ishida, Physics Procedia 75, 405 (2015).

${ }^{39}$ M. S. Petrushevsky, P. K. Rout, G. Levi, A. Kohn, and Y. Dagan, Phys. Rev. B 95, 085112 (2017).

${ }^{40}$ J. Yong et al., Appl. Phys. Lett. 105, 222403 (2014).

${ }^{41}$ S. Lee et al., Phys. Rev. X 6, 031031 (2016).

${ }^{42}$ Y. F. Li, Q. L. Ma, S. X. Huang, and C. L. Chien, Sci. Adv. 4, eaap8294 (2018).

${ }^{43}$ E. Saitoh et al., Appl. Phys. Lett. 88, 182509 (2006).

${ }^{44}$ K. Ando et al., J. Appl. Phys. 109, 103913 (2011).

${ }^{45}$ W. Zhang, et al. J. Appl. Phys. 117, 172610 (2015).

${ }^{46}$ J. C. Rojas-Sánchez et al., Phys. Rev. Lett. 116, 096602 (2016).

${ }^{47}$ O. Mosendz et al., Phys. Rev. Lett. 104, 046601 (2010).

${ }^{48}$ Z. Y. Qiu et al., Nat. Commun. 7, 12670 (2016).

${ }^{49}$ E. Shigematsu et al., Appl. Phys. Express 9, 053002 (2016).

${ }^{50}$ H. L. Wang et al., Phys. Rev. Lett. 112, 197201 (2014).

${ }^{51}$ D. Qu, S. Y. Huang, B. F. Miao, S. X. Huang, and C. L. Chien, Phys. Rev. B 89, 140407 (2014).

52 The details about the samples information are provided on pages 1-2 of the Supplementary Information, which includes Ref. 53.

${ }^{53}$ W. Waldhauser, C. Mitterer, J. Laimer, and H. Stroi, Surf. Coat. Technol. 98, 1315 (1998).

${ }^{54}$ M. Z. Hasan, and C. L. Kane, Rev. Mod. Phys. 82, 3045 (2010).

${ }^{55}$ H. Wang et al., Phys. Rev. Lett. 117, 076601 (2016).

${ }^{56}$ V. F. Dmitriev, and B. A. Kalinikos, Sov. Phys. J. 31, 875 (1988).

${ }^{57}$ B. A. Kalinikos, and A. N. Slavin, J. Phys. C 19, 7013 (1986).

${ }^{58}$ A. A. Semenov, et al., Appl. Phys. Lett. 88, 033503 (2006).

${ }^{59}$ The reasons for this observation are provided on page 2 in the Supplementary Information.

${ }^{60}$ N. W. Ashcroft, and N. D. Mermin, Solid State Physics 253-258 (Saunders College, 1976).

${ }^{61}$ C. J. Brower, and C. E. Patton, J. Appl. Phys. 53, 2104 (1982).

${ }^{62}$ D. D. Stancil, and A. Prabhakar, Spin waves-theory and applications (Springer, New York, 2009).

${ }^{63}$ A. G. Gurevich, and G. A. Melkov, Magnetization oscillations and waves (CRC, New York, 1996).

${ }^{64}$ The constant $C$ in Eq. (2) is generally proportional to the real part of the spin mixing conductance at the interface, the frequency of the precession in the ferromagnet, and the square of the sine function of the precession angle. For the fitting shown in Figs. 3(c) and 3(d), one assumes that at a given temperature $C$ is the same in all nine samples.

65 The details about the calculation of skin depth in $\mathrm{SmB}_{6}$ are provided on pages 3-4 in the Supplementary Information, which includes Ref. 66.

${ }^{66}$ Y. Sun et al., Phys. Rev. Lett. 111, 106601 (2013).

${ }^{67}$ J. C. Nickerson et al., Phys. Rev. B 3, 2030 (1971).

${ }^{68}$ J. W. Allen, B. Batlogg, and P. Wachter, Phys. Rev. B 20, 4807 (1979).

${ }^{69}$ S. Wolgast et al., Phys. Rev. B 88, 180405 (2013). 
${ }^{70}$ The details about the theoretical analysis are provided on pages 6-9 in the Supplementary Information, which including the Refs. 71 and 72.

${ }^{71}$ P. P. Baruselli, and M. Vojta, 2D Mater. 2, 044011 (2015).

${ }_{72}^{72}$ P. P. Baruselli, \& M. Vojta, Phy. Rev. B 90, 201106(R) (2014).

${ }^{73}$ Y. Zhang et al., Nat. Phys. 6, 584 (2010).

${ }^{74}$ M. Neupane et al., Nat. Commun. 5, 3841 (2014).

${ }^{75}$ Q. Song et al., Nat. Commun. 7, 13485 (2016).

76 This work was primarily supported by the SHINES, an Energy Frontier Research Center funded by the U.S. Department of Energy, Office of Science, Basic Energy Sciences under Award SC0012670. In addition, the work at CSU was also supported by the C-SPIN, one of the SRC STARnet Centers sponsored by MARCO and DARPA, the U.S. National Science Foundation under Grants No. EFMA-1641989 and No. DMR-1407962, and the U.S. Army Research Office under Grant No. W911NF-14-1-0501; the work at Argonne was supported by the U.S. Department of Energy, Office of Science, Materials Science and Engineering Division. 